\title{
Effects of combined tiotropium/olodaterol on inspiratory capacity and exercise endurance in COPD
}

\author{
Denis E. O'Donnell ${ }^{1}$, Richard Casaburi ${ }^{2}$, Peter Frith ${ }^{3}$, Anne Kirsten ${ }^{4}$, \\ Dorothy De Sousa ${ }^{5}$, Alan Hamilton ${ }^{5}$, Wenqiong $X \mathrm{e}^{6}$ and François Maltais ${ }^{7}$
}

Affiliations: 'Division of Respiratory and Critical Care Medicine, Respiratory Investigation Unit, Queen's University and Kingston General Hospital, Kingston, ON, Canada. ${ }^{2}$ Los Angeles Biomedical Research Institute at Harbor-UCLA Medical Center, Los Angeles, CA, USA. ${ }^{3}$ Respiratory Medicine, Southern Adelaide Local Health Network, Adelaide, Australia. ${ }^{4}$ Pulmonary Research Institute at Lung Clinic Grosshansdorf, Airway Research Center North, Member of the German Center for Lung Research, Grosshansdorf, Germany. ${ }^{5}$ Boehringer Ingelheim, Burlington, ON, Canada. ${ }^{6}$ Boehringer Ingelheim Pharmaceuticals, Ridgefield, CT, USA. ${ }^{7}$ Centre de Recherche, Institut Universitaire de Cardiologie et de Pneumologie de Québec, Université Laval, Québec, QC, Canada.

Correspondence: Denis O'Donnell, Division of Respiratory and Critical Care Medicine, Respiratory Investigation Unit, Queen's University and Kingston General Hospital, 102 Stuart Street, Kingston, K7L 3N6 Ontario, Canada. E-mail: odonnelldaqueensu.ca

@ERSpublications

$\mathrm{T} / \mathrm{O}$ reduces lung hyperinflation in COPD versus $\mathrm{T}, \mathrm{O}$ or placebo and increases exercise endurance versus placebo http://ow.ly/ml3G307XW6a

Cite this article as: O'Donnell DE, Casaburi R, Frith $\mathrm{P}$, et al. Effects of combined tiotropium/olodaterol on inspiratory capacity and exercise endurance in COPD. Eur Respir J 2017; 49: 1601348 [https://doi.org/ 10.1183/13993003.01348-2016].

ABSTRACT Two replicate, double-blind, 6-week, incomplete-crossover studies (MORACTO 1 and 2) assessed the effects of tiotropium/olodaterol on inspiratory capacity and exercise endurance time in patients with moderate to severe chronic obstructive pulmonary disease.

For each patient, four of five treatments were administered once daily for 6 weeks, with a 21-day washout between treatments: tiotropium/olodaterol $2.5 / 5 \mu \mathrm{g}$ or $5 / 5 \mu \mathrm{g}$, tiotropium $5 \mu \mathrm{g}$, olodaterol $5 \mu \mathrm{g}$ or placebo, all via the Respimat inhaler. Primary outcomes were inspiratory capacity prior to exercise and exercise endurance time during constant work-rate cycle ergometry to symptom limitation at $75 \%$ of peak incremental work rate after 6 weeks ( $2 \mathrm{~h}$ post-dose).

295 and 291 patients were treated in MORACTO 1 and 2, respectively. Tiotropium/olodaterol 2.5/5 and $5 / 5 \mu \mathrm{g}$ provided significant improvements in inspiratory capacity versus placebo and monotherapies $(p<0.0001)$, and significant improvements in exercise endurance time versus placebo $(p<0.0001)$. Intensity of breathing discomfort was reduced following both doses of tiotropium/olodaterol versus placebo $(\mathrm{p}<0.0001)$.

Once-daily tiotropium/olodaterol yielded improvements in lung hyperinflation versus placebo and statistically significant improvements versus monotherapies. Tiotropium/olodaterol also showed improvements in dyspnoea and exercise tolerance versus placebo but not consistently versus monotherapies.

This article has supplementary material available from erj.ersjournals.com

Received: July 072016 | Accepted after revision: Dec 142016

Clinical trials: These studies are registered at www.clinicaltrials.gov with identifier numbers NCT01533922 and NCT01533935, and Boehringer Ingelheim study numbers 1237.13 and 1237.14.

Support statement: This work was supported by Boehringer Ingelheim Pharma GmbH \& Co. KG (Ingelheim, Germany). Funding information for this article has been deposited with the Open Funder Registry.

Conflict of interest: Disclosures can be found alongside this article at erj.ersjournals.com

Copyright OERS 2017. This ERJ Open article is open access and distributed under the terms of the Creative Commons Attribution Non-Commercial Licence 4.0. 


\section{Introduction}

Activity-related breathlessness caused by dynamic hyperinflation is a characteristic feature of chronic obstructive pulmonary disease (COPD) that limits exercise tolerance and impacts on activities of daily living, leading to physical deconditioning and reduced quality of life [1-5]. Improving dyspnoea and exercise tolerance are now recognised as important goals in the treatment of stable COPD [6], with the measurement of exercise endurance considered a valuable component of COPD assessment, particularly in response to treatment interventions [7,8]. Current trials of COPD treatments use a variety of exercise tests, such as the 6-min walk test, incremental and endurance shuttle walk tests, and cycle ergometry and treadmill tests [9]. In particular, constant work-rate cycle ergometry (CWRCE) is now often used in efficacy evaluations of long-acting bronchodilators because it allows a thorough evaluation of the physiological response during exercise and due to its documented responsiveness to treatment $[8,10]$.

Exercise studies have provided insights into the mechanistic factors responsible for reductions in exertional breathlessness and improvements in symptom-limited exercise endurance in COPD following bronchodilator treatment [11]. These improvements include sustained lung volume reduction as a result of improved tidal expiratory flow rates and lung emptying, with reduced resting and exercise lung hyperinflation and a delay in the mechanical limitation to ventilation [12]. Consequently, exertional dyspnoea is alleviated, leading to increases in exercise endurance time (EET).

There is a large body of evidence that the long-acting muscarinic antagonist (LAMA) tiotropium can increase inspiratory capacity, reduce exertional breathlessness and improve EET in patients with COPD $[11,13-18]$. The long-acting $\beta_{2}$-agonist (LABA) olodaterol is approved as a once-daily maintenance treatment for patients with COPD [19-21], and has been shown in two, 6-week, crossover studies to be effective in improving inspiratory capacity, reducing exertional breathlessness and increasing EET in patients with moderate to very severe COPD [22].

The pivotal 52-week TONADO studies demonstrated the bronchodilating efficacy of maintenance treatment with tiotropium/olodaterol $2.5 / 5 \mu \mathrm{g}$ and $5 / 5 \mu \mathrm{g}$ in patients with moderate to very severe COPD, with improvements compared with tiotropium and olodaterol monotherapies with respect to airflow limitation (assessed via forced expiratory volume in $1 \mathrm{~s}$ (FEV1)), health-related quality of life (assessed via the St George's Respiratory Questionnaire) and dyspnoea during daily activities (assessed via the Transition Dyspnoea Index) [23]. The MORACTO studies further extended the evaluation of the efficacy of tiotropium/olodaterol by investigating whether the effects on lung hyperinflation, exertional dyspnoea and exercise tolerance seen with tiotropium and olodaterol monotherapies are maintained, or even extended, when the two therapies are combined.

The primary objective of the MORACTO studies was to investigate the effects of 6 weeks of treatment with tiotropium/olodaterol compared with placebo, tiotropium and olodaterol on inspiratory capacity and EET during CWRCE in patients with moderate to severe COPD.

\section{Methods}

Patients

Male or female patients (aged 40-75 years) were enrolled if they met the following inclusion criteria: post-bronchodilator (400 $\mu$ g salbutamol) $\mathrm{FEV} 1 /$ forced vital capacity (FVC) $<70 \%$; post-bronchodilator $\mathrm{FEV} 1$ $\geqslant 30 \%$ and $<80 \%$ of predicted normal (Global Initiative for Chronic Obstructive Lung Disease (GOLD) $2-3$ ); current or ex-smokers with a smoking history of $>10$ pack-years. To ensure a broadly representative COPD patient population, the presence of static lung hyperinflation (such as increased functional residual capacity (FRC)) was not an entry requirement. Key exclusion criteria included: significant disease other than COPD; unstable or life-threatening cardiac arrhythmia; hospitalisation for heart failure or myocardial infarction within the past year; regular use of daytime oxygen therapy for $>1 \mathrm{~h}$ per day; history of asthma and contraindications to exercise as per the European Respiratory Society guidelines [24].

\section{Study design}

MORACTO 1 (Boehringer Ingelheim study 1237.13; ClinicalTrials.gov trial NCT01533922) and 2 (Boehringer Ingelheim study 1237.14; ClinicalTrials.gov trial NCT01533935) were replicate, 6-week, randomised, double-blind, placebo-controlled, five-treatment, four-period, incomplete-crossover, phase III studies (figure 1). Following an initial screening visit, there was a 2 - to 4 -week run-in period to ensure clinical stability and perform baseline assessments. During this screening period, patients completed an incremental work-rate cycle ergometry test, a training CWRCE test and a baseline CWRCE test. Patients with an EET $\geqslant 25$ min during the training or baseline CWRCE tests were excluded from the study. Patients were randomised at visit 3 into the double-blind, crossover treatment portion of the trial in which they received four of the five possible treatments once daily for 6 weeks: tiotropium/olodaterol $2.5 / 5 \mu \mathrm{g}$ or $5 / 5 \mu \mathrm{g}$, tiotropium $5 \mu \mathrm{g}$, olodaterol $5 \mu \mathrm{g}$ or placebo, all via the Respimat inhaler (Boehringer Ingelheim 


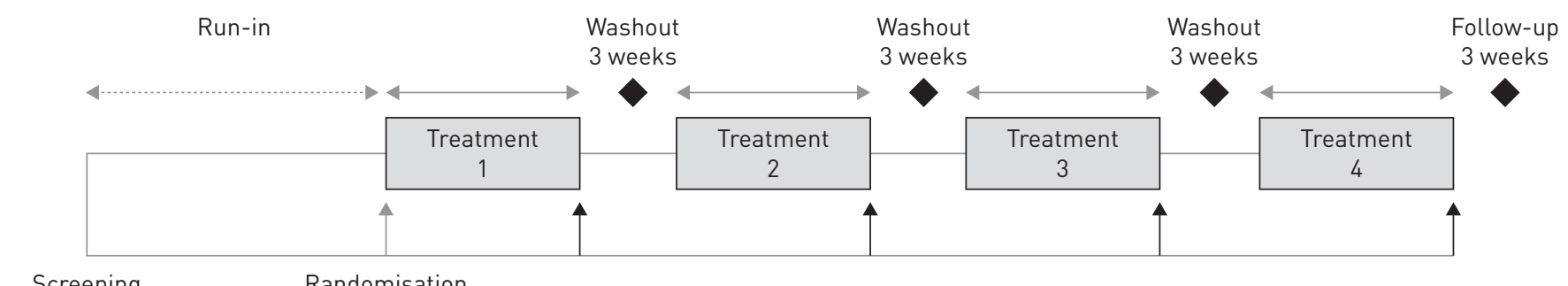

Screening

Randomisation

$\longleftrightarrow 6$ weeks

$4 \ldots . .-4$ weeks
Baseline values: CWRCE; Borg scale; IC
A Primary end-points: IC pre-exercise (2 $\mathrm{h}$ post-dose) and EET during CWRCE Secondary end-points: Borg scale

FIGURE 1 Study design. 0: olodaterol; T: tiotropium; CWRCE: constant work-rate cycle ergometry; IC: inspiratory capacity; EET: exercise endurance time. Incomplete-crossover study: four of five treatments given once daily in random order (placebo, $05 \mu \mathrm{g}, \mathrm{T} 5 \mu \mathrm{g}, \mathrm{T} / 02.5 / 5 \mu \mathrm{g}$ or $\mathrm{T} / 05 / 5 \mu \mathrm{g}$ ), all via the Respimat inhaler.

Pharma GmbH \& Co. KG, Ingelheim, Germany). Patients were randomly assigned to one of five possible treatment sequences. Each treatment period was separated by a 21-day washout and patients were followed-up for 21 days after completion of the last treatment period or, in the case of early discontinuation, after the final dose of study medication.

\section{Exercise testing}

Incremental cycle ergometry to symptom limitation was performed at screening ( $10 \mathrm{~W}$ increments. $\left.\mathrm{min}^{-1}\right)$ to determine peak work rate, defined as the highest work rate maintained for $\geqslant 30 \mathrm{~s}$. All subsequent symptom-limited CWRCE tests were conducted at $75 \%$ of peak work rate. Prior to randomisation, two CWRCE tests were performed: the first was included as a "training" test, while the second represented the pre-treatment baseline test. Following randomisation, CWRCE tests were also conducted at the end of each treatment period, $2 \mathrm{~h}$ after inhalation of study medication. Inspiratory capacity and intensity of breathing discomfort and leg discomfort (standardised version of the modified Borg scale [25]) were measured prior to exercise, every 2 min during exercise and at the point of symptom limitation (end-exercise). Isotime was defined for each patient as the shortest exercise duration observed in all constant work-rate tests (baseline and all treatment periods). Heart rate and blood pressure measurements were also recorded during exercise and reported as 30-s averages. After completing each exercise test, patients identified the primary reason for stopping (due to leg and/or breathing discomfort or other reason).

The studies were carried out in accordance with the principles of the Declaration of Helsinki and the International Conference on Harmonisation Harmonised Tripartite Guideline for Good Clinical Practice; written informed consent was obtained from each patient.

\section{End-points}

The primary efficacy end-points were assessed after 6 weeks of treatment: inspiratory capacity prior to exercise ( $2 \mathrm{~h}$ post-dose, immediately prior to CWRCE) and EET during CWRCE. Secondary end-points were also assessed after 6 weeks of treatment and included Borg-time slope for intensity of breathing discomfort during CWRCE. The Borg-time slope was calculated as the intensity of breathing discomfort at end-exercise minus the intensity of breathing discomfort at pre-exercise, divided by EET. Further end-points included FVC $1 \mathrm{~h}$ post-dose, isotime and exercise values for inspiratory capacity, and intensity of breathing discomfort and leg discomfort (Borg scale).

\section{Statistical analysis}

A mixed-effects model repeated measures (MMRM) approach, with treatment, period and study baseline as fixed effects and patient as a random effect, was used for the primary analysis. EET(s) was transformed using $\log _{10}$ to correct for skewness in the original scale and back-transformed for presentation. Study baseline inspiratory capacity prior to exercise and $\log _{10}$-transformed EET at study baseline were added to the MMRM as covariates for the primary end-points.

The primary analysis was conducted on the full analysis set, which included all patients with baseline and any evaluable post-dose primary end-point data. The slope of breathing discomfort was analysed using the same MMRM approach.

Tiotropium/olodaterol combination was evaluated versus comparators as part of a hierarchical testing scheme (including 12 hypothesis tests) with the following fixed sequence: adjusted mean inspiratory capacity for 
tiotropium/olodaterol versus placebo, then versus the respective monotherapies followed by mean EET (adjusted for study baseline) for tiotropium/olodaterol versus placebo, then versus monotherapies. Full details of this sequence are included in supplementary figure S1. Statistical significance was declared if a hypothesis test and all previous hypothesis tests in the hierarchy were significant at the two-sided 0.05 level. In the context of comparisons involving multiple statistical tests, the p-value of each test is called the nominal p-value.

Safety was summarised descriptively. Further details of methodology and statistical testing are included in the supplementary material.

\section{Results}

Each study was conducted independently; however, because of their identical design and for ease of reading, we present the combined results of MORACTO 1 and 2 (individual trial data are included in the supplementary material). A total of 586 patients were randomised and treated in 82 investigational sites in 13 countries (figure 2). Overall, 571 patients were included in the full analysis set; the majority (85.5\%) completed all four treatment periods. Discontinuation rates were $<5 \%$ and similar across treatment groups (figure 2 and supplementary figure S2).

Baseline patient characteristics, including exercise-specific characteristics, are shown in table 1 [26]. The majority of patients were male, with a mean age of $\sim 62$ years and a mean body mass index of $27 \mathrm{~kg} \cdot \mathrm{m}^{-2}$. Most patients $(\sim 70 \%)$ were GOLD 2, with $\sim 30 \%$ GOLD 3. Baseline patient characteristics for the individual studies are shown in supplementary table S1.

\section{Efficacy}

\section{Inspiratory capacity}

After 6 weeks of once-daily treatment with tiotropium/olodaterol $2.5 / 5$ or $5 / 5 \mu \mathrm{g}$, there was a statistically significant increase in inspiratory capacity prior to exercise ( $2 \mathrm{~h}$ post-dose) of 245 and $254 \mathrm{~mL}(\mathrm{p}<0.0001)$ compared with placebo, 90 and $99 \mathrm{~mL}(\mathrm{p}<0.0001)$ compared with olodaterol, and 92 and $101 \mathrm{~mL}$ compared with tiotropium $(\mathrm{p}<0.0001)$, respectively (table 2$)$. The improvements in inspiratory capacity with tiotropium/olodaterol $2.5 / 5$ or $5 / 5 \mu \mathrm{g}$ compared with placebo and monotherapies were sustained during exercise at both isotime and end-exercise (figure 3). Similar results were seen in the individual studies (supplementary table S2 and supplementary figure S3). There was a significantly greater proportion of patients who were classified as inspiratory capacity responders with both tiotropium/olodaterol doses compared with placebo and monotherapies (supplementary figure S4).

\section{EET during CWRCE}

There was a statistically significant increase in mean EET after 6 weeks of treatment with tiotropium/ olodaterol $2.5 / 5$ and $5 / 5 \mu \mathrm{g}$ compared with placebo (19.2\% and $17.3 \%$, respectively; $\mathrm{p}<0.0001$ ) (figure 4). Similar responses were seen in the individual studies (supplementary figure S5).

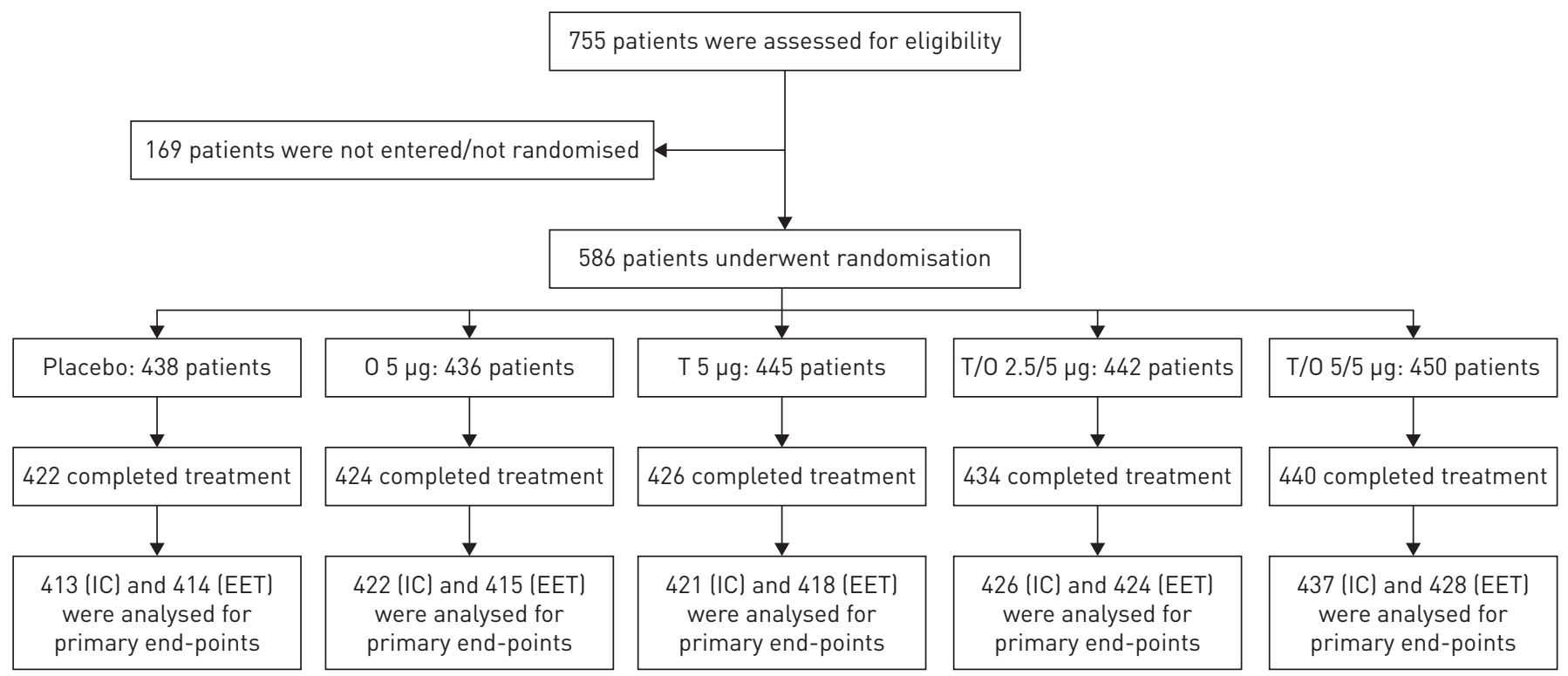

FIGURE 2 Participant flow (combined studies). O: olodaterol; T: tiotropium; IC: inspiratory capacity; EET: exercise endurance time. As these were incomplete-crossover studies, patients received four out of five possible treatments. 


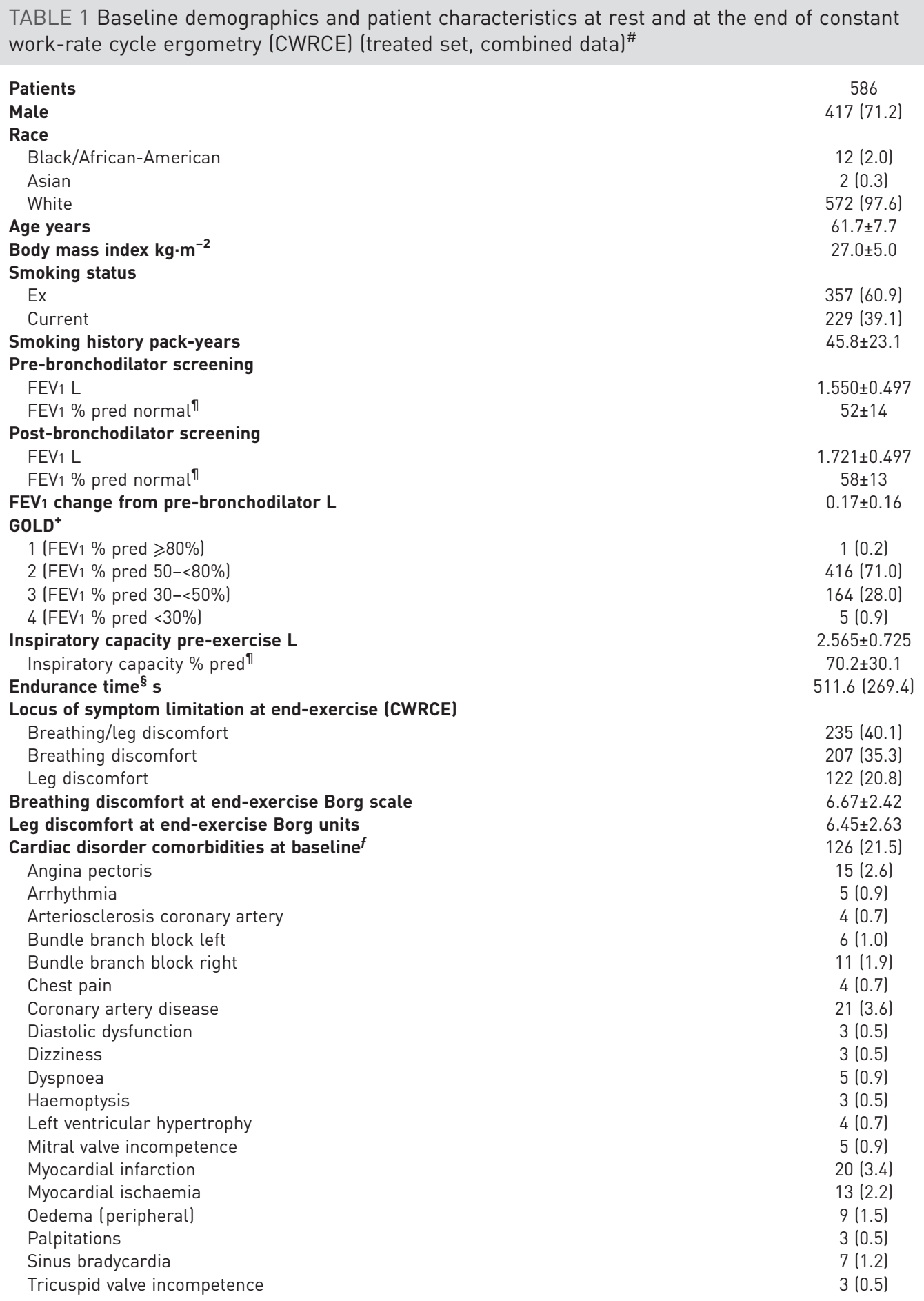

Data are presented as $\mathrm{n}, \mathrm{n}(\%)$ or mean \pm SD. $F E V 1$ : forced expiratory volume in $1 \mathrm{~s}$; GOLD: Global Initiative for Chronic Obstructive Lung Disease. ${ }^{\#}$ : individual study data are presented in supplementary table $\mathrm{S} 1$; I: based on predicted values defined by the European Community for Steel and Coal [26]. ${ }^{+}$: one patient with GOLD 1 disease was randomised to treatment (this was classed as a protocol violation and the patient was excluded from the per-protocol analysis set); five patients with GOLD 4 disease were randomised to treatment: two patients were enrolled prior to a protocol amendment that excluded patients with GOLD 4 disease (the original protocol did not include a lower limit for $\mathrm{FEV}_{1} \%$ pred) and one patient had FEV $1 \%$ pred of $29.77 \%$; these were not considered protocol violations; two patients with GOLD 4 disease were randomised after approval of the protocol amendment and were excluded from the per-protocol analysis set; ${ }^{\S}$ : arithmetic mean (not log-transformed); ${ }^{f}$ : coded according to the Medical Dictionary for Regulatory Activities version 16.1 (www.meddra.org) and occurring in $\geqslant 0.5 \%$ of patients at baseline. 
TABLE 2 Adjusted ${ }^{\#}$ mean inspiratory capacity pre-exercise: treatment comparisons after 6 weeks of treatment (full analysis set, combined data)

\begin{tabular}{|c|c|c|}
\hline Treatment comparison & Adjusted inspiratory capacity L $(95 \% \mathrm{Cl})$ & p-value \\
\hline Common baseline & $2.561 \pm 0.030$ & \\
\hline \multicolumn{3}{|l|}{ Tiotropium/olodaterol $2.5 / 5 \mu \mathrm{g}$} \\
\hline versus placebo & $0.245 \pm 0.019(0.209-0.282)$ & $<0.0001$ \\
\hline versus olodaterol $5 \mu \mathrm{g}$ & $0.090 \pm 0.019(0.054-0.127)$ & $<0.0001$ \\
\hline versus tiotropium $5 \mu \mathrm{g}$ & $0.092 \pm 0.019(0.055-0.128)$ & $<0.0001$ \\
\hline \multicolumn{3}{|l|}{ Tiotropium/olodaterol $5 / 5 \mu \mathrm{g}$} \\
\hline versus placebo & $0.254 \pm 0.019(0.218-0.291)$ & $<0.0001$ \\
\hline versus olodaterol $5 \mu \mathrm{g}$ & $0.099 \pm 0.018(0.063-0.136)$ & $<0.0001$ \\
\hline versus tiotropium $5 \mu \mathrm{g}$ & $0.101 \pm 0.018(0.065-0.137)$ & $<0.0001$ \\
\hline \multicolumn{3}{|c|}{$\begin{array}{l}\text { Data are presented as mean } \pm \mathrm{SD} \text {, unless otherwise stated. }{ }^{\#} \text { : adjusted mean difference obtained from the } \\
\text { mixed-effects model repeated measures approach with fixed effects of treatment and period, study } \\
\text { baseline as covariate, patient as a random effect, and compound symmetry as a covariance structure for } \\
\text { within-patient variation. }\end{array}$} \\
\hline
\end{tabular}

The increase in EET for both doses of tiotropium/olodaterol compared with olodaterol $5 \mu \mathrm{g}$ reached nominal statistical significance $(\mathrm{p}<0.01$ for tiotropium/olodaterol $2.5 / 5 \mu \mathrm{g} ; \mathrm{p}<0.05$ for tiotropium/ olodaterol $5 / 5 \mu \mathrm{g}$ ), with numerical increases in EET compared with tiotropium $5 \mu \mathrm{g}$ (figure 4). Results slightly differed between the individual studies: numerical increases in EET were observed only with tiotropium/olodaterol 2.5/5 $\mu \mathrm{g}$ compared with either monotherapy in MORACTO 1, while statistically significant increases in EET for both doses of tiotropium/olodaterol were seen in MORACTO 2 versus olodaterol $5 \mu \mathrm{g}(\mathrm{p}<0.05)$ but not tiotropium $5 \mu \mathrm{g}$ (supplementary figure S5).

There was a significantly greater proportion of patients who were classified as EET responders with both tiotropium/olodaterol doses compared with placebo (supplementary figures S6 and S7).

\section{Breathing discomfort}

There was a reduction in breathing discomfort for tiotropium/olodaterol versus placebo as indicated by nominally significant reductions in both the slope and intensity of breathing discomfort at isotime

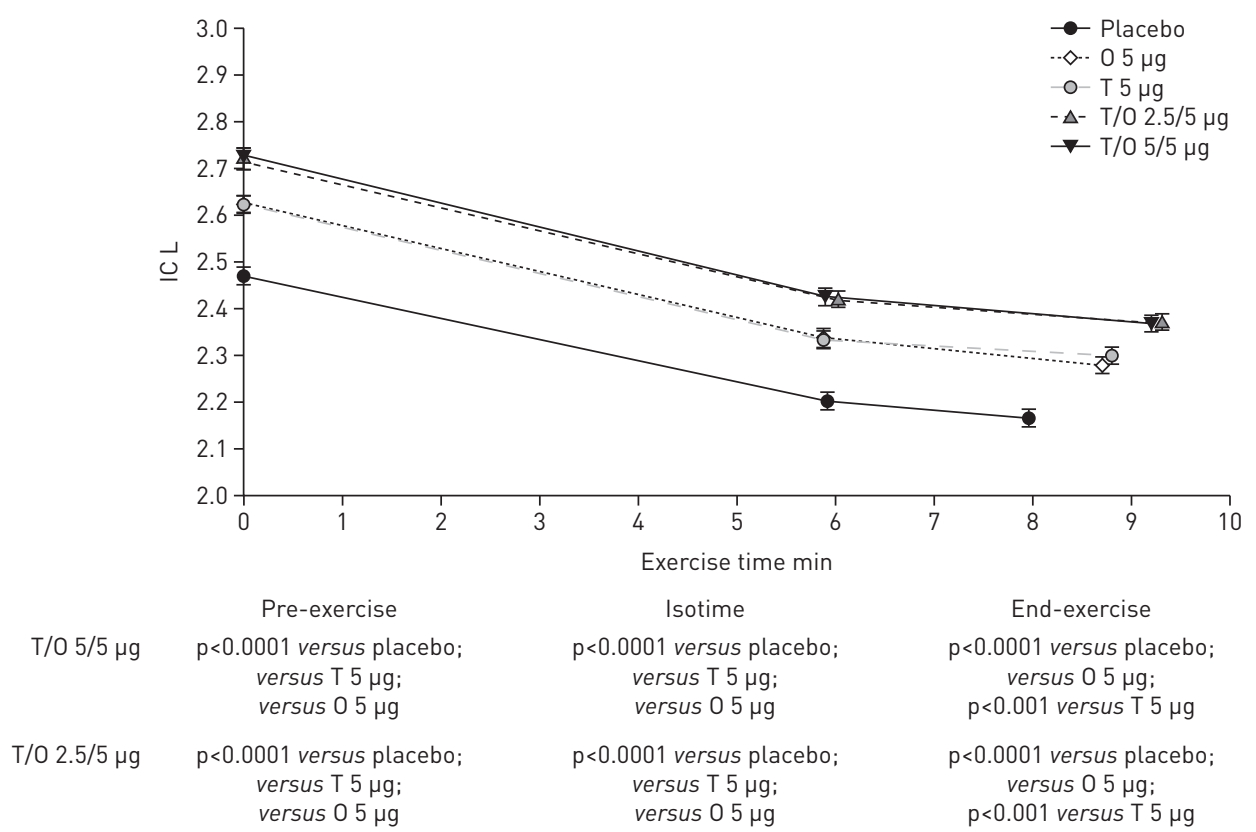

FIGURE 3 Adjusted mean \pm SE inspiratory capacity (IC) pre-exercise, at isotime and at end-exercise after 6 weeks (combined studies). O: olodaterol; T: tiotropium. As each patient only received four out of five possible treatments, isotimes differ between treatments. 


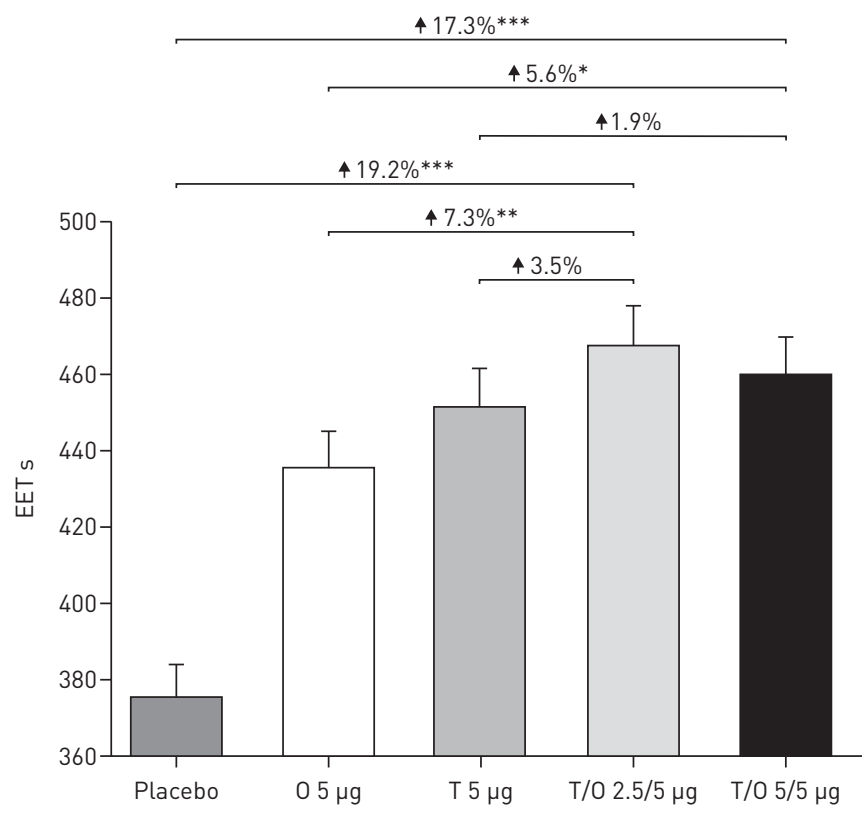

FIGURE 4 Adjusted geometric mean \pm SE exercise endurance time (EET) during constant work-rate cycle ergometry after 6 weeks of treatment (combined studies). 0 : olodaterol; T: tiotropium. ${ }^{*}: p<0.05$; ${ }^{* *}: p<0.01$; $* * *: p<0.0001$

(figure 5), with similar results seen in the individual studies (supplementary table S3 and supplementary figure S8). Tiotropium/olodaterol $2.5 / 5$ and $5 / 5 \mu$ g reduced breathing discomfort compared with placebo at isotime by 0.740 and 0.693 Borg units, respectively $(\mathrm{p}<0.0001)$. Comparisons between tiotropium/ olodaterol and the monotherapies with respect to breathing discomfort followed a similar pattern to EET, with a trend towards a reduction in breathing discomfort compared with olodaterol but similar reductions in breathing discomfort for tiotropium/olodaterol compared with tiotropium (figure 5).

\section{Additional end-points}

Spirometry parameters (FEV1, FVC) increased following treatment with tiotropium/olodaterol compared with placebo and monotherapies; details are included in supplementary tables S4 and S5.

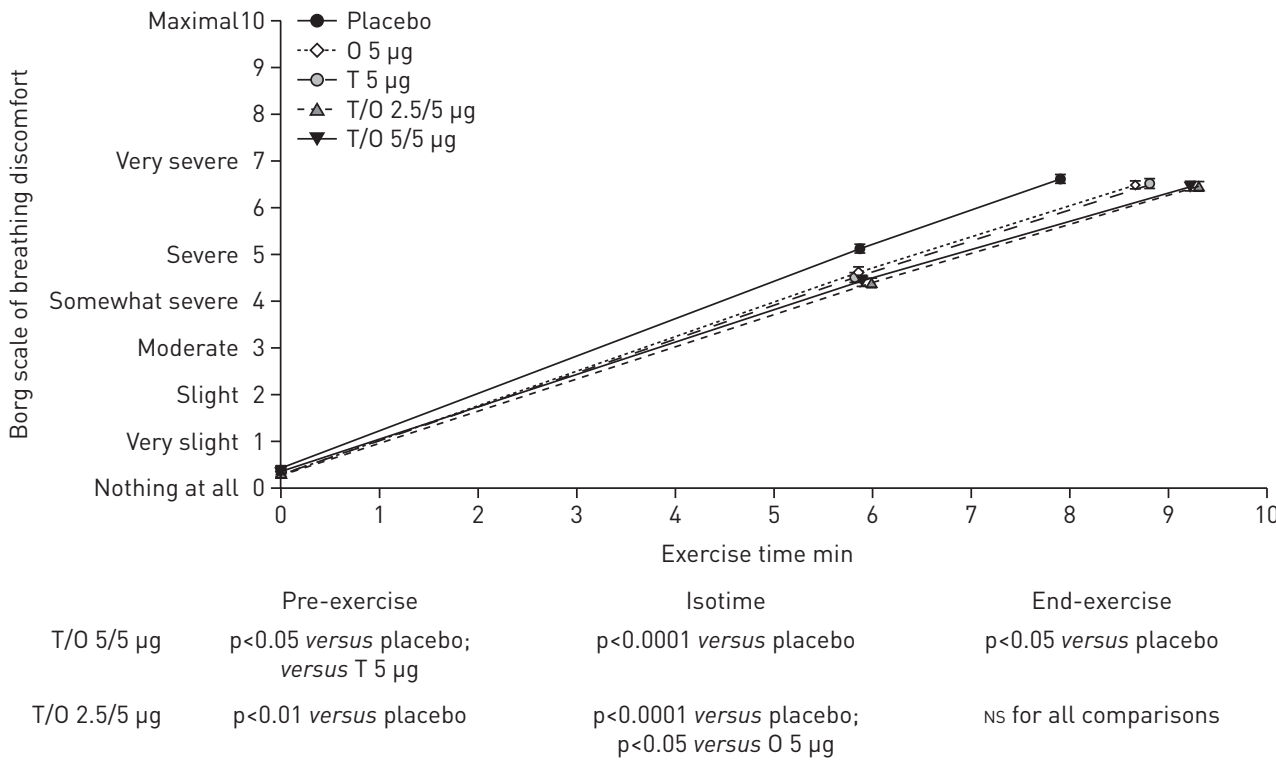

FIGURE 5 Adjusted mean \pm SE intensity of breathing discomfort (Borg scale) after 6 weeks at pre-exercise, isotime and end-exercise (combined studies). 0: olodaterol; T: tiotropium. NS: nonsignificant. 
Safety and tolerability

Incidences of adverse events, severe adverse events and adverse events leading to discontinuations were similar to placebo for all treatments (supplementary table S6). There were no clinically relevant changes in laboratory parameters, vital signs or ECG parameters in either study.

\section{Discussion}

Results from these two replicate studies demonstrate that tiotropium/olodaterol reduces lung hyperinflation prior to and during exercise in patients with COPD compared with placebo and monotherapies. The primary end-point of inspiratory capacity prior to exercise was met, with significant increases with tiotropium/olodaterol $2.5 / 5$ and $5 / 5 \mu \mathrm{g}$ compared with tiotropium $5 \mu \mathrm{g}$, olodaterol $5 \mu \mathrm{g}$ and placebo. Additional serial assessments of inspiratory capacity during exercise demonstrated that these improvements were maintained at isotime and end-exercise. Improvements in inspiratory capacity were accompanied by significantly longer EET with both doses of tiotropium/olodaterol compared with placebo. However, despite statistically significant increases in inspiratory capacity for tiotropium/olodaterol versus tiotropium or olodaterol monotherapy, increases in EET with tiotropium/olodaterol were not consistently demonstrated compared with the monotherapies. Post hoc analyses did not identify any specific subgroup of patients exhibiting greater improvements in EET with tiotropium/olodaterol versus monotherapy; details of these post hoc analyses are provided in the supplementary material. As previously reported [27], endurance time during constant work-rate exercise is asymmetrically distributed around the mean, with a significant skew towards long EETs. Therefore, in contrast to previous trials of cycling endurance time in COPD, statistical analyses were performed on $\log _{10}$-transformed endurance time data. However, in order to facilitate comparisons with previous studies, we also report the arithmetic mean treatment effects (supplementary table S7).

Exercise studies such as MORACTO have now become standard in many clinical programmes in COPD to gain a more comprehensive appreciation of the efficacy profile of new therapeutics. Two replicate studies were conducted for independent substantiation of the results. In addition, to be consistent with other studies in the phase III programme, two doses of tiotropium/olodaterol were investigated in the MORACTO studies. Finally, in contrast to early bronchodilator exercise studies $[11,28]$, evidence of lung hyperinflation (e.g. FRC \% pred $>120 \%$ ) was not an inclusion criterion in the MORACTO studies, as the intention was to enrol a study population that was broadly similar to other studies in the phase III programme. In line with a previous study of the effects of tiotropium on lung hyperinflation, dyspnoea and exercise tolerance, patients with exercise limitation related to factors other than fatigue or exertional dyspnoea were excluded from this study and there were no other protocol differences of note [11].

The inspiratory capacity results prior to and during exercise in MORACTO complement the results from the VIVACITO body plethysmography substudy [29], which evaluated the effects of tiotropium/olodaterol 2.5/5 and $5 / 5 \mu \mathrm{g}$ on lung volume parameters (FRC, residual volume, total lung capacity (TLC), inspiratory capacity) at rest in patients with COPD. In the VIVACITO substudy, after 6 weeks of once-daily treatment, tiotropium/olodaterol $2.5 / 5$ and $5 / 5 \mu \mathrm{g}$ increased inspiratory capacity (TLC-FRC) by 0.305 and $0.351 \mathrm{~L}$, respectively. Consistent with VIVACITO, in MORACTO, inspiratory capacity increased at week 6 by 0.218 $0.274 \mathrm{~L}$ with tiotropium/olodaterol $(2.5 / 5$ and $5 / 5 \mu \mathrm{g})$ compared with placebo. There is robust evidence that long-acting bronchodilator (LAMA, LABA) maintenance therapy in patients with COPD provides sustained lung volume reduction as a result of improved tidal expiratory flow rates and lung emptying, with reduced resting and exercise lung hyperinflation, and a delay in the mechanical limitation to ventilation, with a consequent alleviation of exertional dyspnoea and an increase in symptom-limited EET $[11,30]$. With the availability of once-daily LAMA/LABA combinations, recent attention has focused on exploring the effects of combination therapy versus placebo and monotherapy on indices of lung hyperinflation, exertional dyspnoea and EET. BEEH et al. [31] investigated the effects of QVA149 (a combination of indacaterol and glycopyrronium) versus placebo and tiotropium in patients with COPD with a post-bronchodilator FEV 1 of $1.6 \mathrm{~L}$ (56\% predicted). Overall, 73\% of patients were GOLD 2 and 27\% were GOLD 3. As with the present studies, the presence of lung hyperinflation was not an inclusion criterion. After 3 weeks of once-daily treatment, the combination demonstrated a statistically significant improvement in inspiratory capacity compared with both placebo and tiotropium pre-exercise, at isotime and at end-exercise. Statistically significant improvements in EET during CWRCE at 75\% of peak work rate were demonstrated compared with placebo; however, as in our studies, there was no significant difference in EET compared with tiotropium [31]. Similar effects were observed in a study assessing the efficacy of umeclidinium/vilanterol in improving EET, using the endurance shuttle walk test, in patients with COPD. Improvements in EET were significant compared with placebo at week 12 in one study but this was not replicated in the parallel study. This was suggested to be due to the unexpectedly high placebo response in the second study [32].

Inspiratory capacity indicates the position of tidal volume relative to TLC and, therefore, its proximity to the upper, less compliant reaches of the respiratory system's static pressure-volume relation. The lower the 
resting inspiratory capacity, the closer the tidal volume is to the TLC and the greater the elastic load on the inspiratory muscles. As such, it is somewhat unexpected that the additional benefits of dual bronchodilation on inspiratory capacity noted by BEEH et al. [31] and in MORACTO did not translate into less exertional dyspnoea and prolonged exercise tolerance. Although we do not have a definitive explanation for this observation, it may well be that in patients with moderate airway obstruction and relatively mild lung hyperinflation, an inspiratory capacity increase of $0.1-0.15 \mathrm{~L}$ during exercise for combination treatment versus monotherapy is not sufficient to have clinically important effects on exertional breathlessness. Also, there could be a limit for bronchodilation to immediately translate into better exercise tolerance. In fact, it may be unrealistic to expect the same exercise benefit when adding a second therapy to an existing one than when adding a therapy to placebo. Providing additional bronchodilation with a second bronchodilator may result in two outcomes during exercise [33]: prolonging exercise duration or shifting the locus of symptom limitation from dyspnoea to leg fatigue [13]. In the latter situation, an improvement in exercise duration with bronchodilation is less likely [34, 35]. In this situation, combining exercise training (to improve limb muscle fatigue) to bronchodilation could produce optimal results [17].

It is noteworthy that the study by ВеEH et al. [31] and the present studies used similar inclusion criteria to those used in the respective clinical programmes. While this was a reasonable approach to maintain consistency with study populations from other trials in the respective clinical programmes, it may have led to limitations in the ability to explore the relative effects of the combinations versus monotherapies by not ensuring that the study population had clear evidence of airflow limitation during exercise, e.g. by requiring evidence of lung hyperinflation and/or activity-related dyspnoea. Future studies that include a patient phenotype with more severe COPD, evidence of resting (and dynamic) hyperinflation at screening plus respiratory symptoms as their primary cause for exercise limitation are warranted. Approximately one-fifth of patients had cardiac disorders at baseline. This may have added an additional confounding factor due to lung-heart interactions following anti-muscarinic and/or $\beta$-agonist stimulus. The study was further complicated by the incomplete-crossover design, whereby patients only received four of five possible treatments. Thus, some patients may have received tiotropium/olodaterol but not tiotropium $5 \mu \mathrm{g}$. Furthermore, the incomplete-crossover design meant that each patient was followed-up over a relatively long period (>30 weeks) during which their condition may have changed.

The lack of a significant difference in breathing discomfort at isotime between tiotropium/olodaterol and tiotropium may be contrasted with the statistically significant reduction in Transition Dyspnoea Index focal score observed in the pivotal TONADO studies, which is indicative of a reduction in the degree of dyspnoea experienced during everyday activities. Furthermore, a statistically significant reduction in rescue medication use for tiotropium/olodaterol compared with monotherapies is also suggestive of incremental benefit for tiotropium/olodaterol over monotherapies in alleviating symptoms. Thus, further studies are warranted to better understand the relationship between lung hyperinflation, exertional dyspnoea and exercise tolerance for combination monotherapy.

\section{Conclusions}

These two replicate studies have extended the evidence of the effects of tiotropium/olodaterol on lung hyperinflation by demonstrating increased inspiratory capacity prior to and during exercise compared with placebo or monotherapies. The studies have also demonstrated that the reductions in lung hyperinflation resulted in clinically important reductions in dyspnoea during exercise and symptom-limited EET for the combination compared with placebo. Further studies are required to better understand the conditions under which reductions in lung hyperinflation for combination bronchodilators versus monotherapies may translate into improvements in breathing discomfort and exercise endurance.

\section{Acknowledgements}

Medical writing assistance was provided by Laura George of Complete HealthVizion (Macclesfield, UK), which was contracted and compensated by Boehringer Ingelheim Pharma GmbH \& Co. KG (Ingelheim, Germany).

The authors meet criteria for authorship as recommended by the International Committee of Medical Journal Editors. They take full responsibility for the scope, direction, content of and editorial decisions relating to the manuscript, were involved at all stages of development, and have approved the submitted manuscript. The authors received no compensation related to the development of the manuscript.

\section{References}

1 ZuWallack R. How are you doing? What are you doing? Differing perspectives in the assessment of individuals with COPD. COPD 2007; 4: 293-297.

2 Jones PW. Activity limitation and quality of life in COPD. COPD 2007; 4: 273-278.

3 O'Donnell DE, Laveneziana P. Dyspnea and activity limitation in COPD: mechanical factors. COPD 2007; 4 : $225-236$. 
4 Garcia-Rio F, Lores V, Mediano O, et al. Daily physical activity in patients with chronic obstructive pulmonary disease is mainly associated with dynamic hyperinflation. Am J Respir Crit Care Med 2009; 180: 506-512.

5 Watz H, Pitta F, Rochester CL, et al. An official European Respiratory Society statement on physical activity in COPD. Eur Respir J 2014; 44: 1521-1537.

6 Global Initiative for Chronic Obstructive Lung Disease. Global strategy for the diagnosis, management, and prevention of chronic obstructive pulmonary disease. 2016. www.goldcopd.org Date last accessed: November 11, 2016.

7 American Thoracic Society, American College of Chest Physicians. ATS/ACCP statement on cardiopulmonary exercise testing. Am J Respir Crit Care Med 2003; 167: 211-277.

8 Borel B, Provencher S, Saey D, et al. Responsiveness of various exercise-testing protocols to therapeutic interventions in COPD. Pulm Med 2013; 2013: 410748

9 Fotheringham I, Meakin G, Punekar YS, et al. Comparison of laboratory- and field-based exercise tests for COPD: a systematic review. Int I Chron Obstruct Pulmon Dis 2015; 10: 625-643.

10 Puente-Maestu L, Palange P, Casaburi R, et al. Use of exercise testing in the evaluation of interventional efficacy: an official ERS statement. Eur Respir J 2016; 47: 429-460.

11 O'Donnell DE, Flüge T, Gerken F, et al. Effects of tiotropium on lung hyperinflation, dyspnoea and exercise tolerance in COPD. Eur Respir J 2004; 23: 832-840.

12 O'Donnell DE, Hamilton AL, Webb KA. Sensory-mechanical relationships during high-intensity, constant-work-rate exercise in COPD. J Appl Physiol 2006; 101: 1025-1035.

13 Maltais F, Hamilton A, Marciniuk D, et al. Improvements in symptom-limited exercise performance over 8 h with once-daily tiotropium in patients with COPD. Chest 2005; 128: 1168-1178.

14 Verkindre C, Bart F, Aguilaniu B, et al. The effect of tiotropium on hyperinflation and exercise capacity in chronic obstructive pulmonary disease. Respiration 2006; 73: 420-427.

15 Bédard M-E, Brouillard C, Pepin V, et al. Tiotropium improves walking endurance in COPD. Eur Respir J 2012; 39: 265-271.

16 Cooper CB, Celli BR, Jardim JR, et al. Treadmill endurance during 2-year treatment with tiotropium in patients with COPD: a randomized trial. Chest 2013; 144: 490-497.

17 Casaburi R, Kukafka D, Cooper CB, et al. Improvement in exercise tolerance with the combination of tiotropium and pulmonary rehabilitation in patients with COPD. Chest 2005; 127: 809-817.

18 Casaburi R, Maltais F, Porszasz J, et al. Effects of tiotropium on hyperinflation and treadmill exercise tolerance in mild to moderate chronic obstructive pulmonary disease. Ann Am Thorac Soc 2014; 11: 1351-1361.

19 Ferguson GT, Feldman GJ, Hofbauer P, et al. Efficacy and safety of olodaterol once daily delivered via RespimatC in patients with GOLD 2-4 COPD: results from two replicate 48-week studies. Int J Chron Obstruct Pulmon Dis 2014; 9: 629-645.

20 Lange P, Aumann J-L, Hamilton A, et al. The 24 hour lung function time profile of olodaterol once daily versus placebo and tiotropium in patients with moderate to very severe chronic obstructive pulmonary disease. $J$ Pulm Respir Med 2014; 4: 196.

21 Koch A, Pizzichini E, Hamilton A, et al. Lung function efficacy and symptomatic benefit of olodaterol once daily delivered via Respimat $\odot$ versus placebo and formoterol twice daily in patients with GOLD 2-4 COPD: results from two replicate 48-week studies. Int J Chron Obstruct Pulmon Dis 2014; 9: 697-714.

22 Maltais F, Kirsten A-M, Hamilton A, et al. Evaluation of the effects of olodaterol on exercise endurance in patients with chronic obstructive pulmonary disease: results from two 6-week crossover studies. Respir Res 2016; 17 : 77.

23 Buhl R, Maltais F, Abrahams R, et al. Tiotropium and olodaterol fixed-dose combination versus mono-components in COPD (GOLD 2-4). Eur Respir J 2015; 45: 969-979.

24 ERS Task Force on Standardization of Clinical Exercise Testing. Clinical exercise testing with reference to lung diseases: indications, standardization and interpretation strategies. Eur Respir J 1997; 10: 2662-2689.

25 Hareendran A, Leidy NK, Monz BU, et al. Proposing a standardized method for evaluating patient report of the intensity of dyspnea during exercise testing in COPD. Int J Chron Obstruct Pulmon Dis 2012; 7: 345-355.

26 Quanjer PH, Tammeling GJ, Cotes JE, et al. Lung volumes and forced ventilatory flows. Eur Respir J 1993; 6: Suppl. 16, 5-40.

27 O'Donnell DE, Travers J, Webb KA, et al. Reliability of ventilatory parameters during cycle ergometry in multicentre trials in COPD. Eur Respir J 2009; 34: 866-874.

28 O'Donnell DE, Voduc N, Fitzpatrick M, et al. Effect of salmeterol on the ventilatory response to exercise in chronic obstructive pulmonary disease. Eur Respir J 2004; 24: 86-94.

29 Beeh K-M, Westerman J, Kirsten A-M, et al. The 24-h lung-function profile of once-daily tiotropium and olodaterol fixed-dose combination in chronic obstructive pulmonary disease. Pulm Pharmacol Ther 2015; 32: 53-59.

30 O'Donnell DE, Casaburi R, Vincken W, et al. Effect of indacaterol on exercise endurance and lung hyperinflation in COPD. Respir Med 2011; 105: 1030-1036.

31 Beeh K-M, Korn S, Beier J, et al. Effect of QVA149 on lung volumes and exercise tolerance in COPD patients: the BRIGHT study. Respir Med 2014; 108: 584-592.

32 Maltais F, Singh S, Donald AC, et al. Effects of a combination of umeclidinium/vilanterol on exercise endurance in patients with chronic obstructive pulmonary disease: two randomized, double-blind clinical trials. Ther Adv Respir Dis 2014; 8: 169-181.

33 Magnussen H, Paggiaro P, Schmidt H, et al. Effect of combination treatment on lung volumes and exercise endurance time in COPD. Respir Med 2012; 106: 1413-1420.

34 Saey D, Debigaré R, Leblanc P, et al. Contractile leg fatigue after cycle exercise. A factor limiting exercise in patients with chronic obstructive pulmonary disease. Am J Respir Crit Care Med 2003; 168: 425-430.

35 Deschênes D, Pepin V, Saey D, et al. Locus of symptom limitation and exercise response to bronchodilation in chronic obstructive pulmonary disease. J Cardiopulm Rehabil Prev 2008; 28: 208-214. 